noch unklar, in welchen Stadien der ALS ein Patient am meisten von diesem Medikament profitiert.

Ein Team des Kings College in London untersuchte die klinischen Daten von 959 ALS-Patienten, die an der ersten Studie zum Dosisbereich von Riluzol teilgenommen hatten. Während dieser Untersuchung wurden die Patienten randomisiert vier Therapiegruppen zugewiesen: $50 \mathrm{mg}$ (237 Patienten), $100 \mathrm{mg}$ (236 Patienten) oder 200 mg (244 Patienten) Riluzol täglich bzw. Placebo. Die Studie wurde zwischen 1992 und 1994 durchgeführt, weswegen die Patienten anhand der heute überholten ElEscorial-Kriterien klassifiziert wurden.

Zur besseren Kategorisierung der Patienten re-analysierte das Team die klinischen Daten und wandte dazu retrospektiv das King's Clinical Staging System an. Anhand dieses Systems wurde die Erkrankung in fünf Stufen eingeteilt, von Stufe 1 (frühes Stadium) bis Stufe 4 (spätes Stadium), wobei Stufe 5 den Tod des Patienten bedeutet. Berücksichtigt werden verschiedene motorische Behinderungen, die für das Fortschreiten der ALS typisch sind, bzw. Ernährungs- und respiratorische Probleme im späten Krankheitsstadium. Basierend auf diesem Schema wurden 355 Patienten in Stufe 2, 451 Patienten in Stufe 3 und 153 Patienten im Stadium 4 in die Studie aufgenommen. Es wurden keine ALS-Patienten der Stufe 1 erfasst.

\section{Wie verlängert Riluzol das Leben von ALS-Patienten?}

Fang T. et al. Stage at which riluzole treatment prolongs survival in patients with amyotrophic lateral sclerosis: a retrospective analysis of data from a dose-ranging study. The Lancet Neurology, 2018. doi: 10.1016/S1474-4422(18)30054-1

Riluzol, ein Glutamat-Antagonist, war das erste Medikament zur Behandlung der Amyotropher Lateralsklerose (ALS). Obwohl seine genaue Wirkung noch nicht bekannt ist, vermutet man einen neuroprotektiven Effekt, und in klinischen Studien ließ sich bereits eine lebensverlängernde Wirkung bei ALS nachweisen. Allerdings war bisher

\section{Ergebnisse}

Wie die Analyse der Daten ergab, verändert Riluzol nicht signifikant die Krankheitsprogression im Vergleich zu Placebo bei Patienten der Stadien 2 und 3. Bei Patienten im Stadium 4 jedoch verlängerte sich die Lebenserwartung unter Riluzol signifikant gegenüber Placebo und zwar vor allem in der Gruppe, die $100 \mathrm{mg}$ Riluzol täglich erhielt. Dort beobachtete man ein um $45 \%$ reduziertes Sterberisiko im Vergleich zu Placebo.

Eine ähnliche Analyse führten die Autoren mit den klinischen Daten durch, die im Verlauf der LiCALS Studie erhoben worden waren. Diese Studie schloss 210 ALS Patienten ein, die randomisiert entweder Lithium oder Placebo erhielten. Wie die Analyse 
dieser Daten ergab, verändert eine Behandlung mit Lithium nicht die Zeitdauer, die ein Patient in einem Stadium verbleibt, verglichen mit Placebo. Das demonstriert, dass der in der Dosisbereichs-Studie beobachtete Effekt eine spezifische Wirkung des Riluzols ist und kein statistisches oder analytisches Artefakt.

FAZIT

Die Ergebnisse deuten darauf hin, dass Riluzol die Überlebensrate von ALS Patienten erhöht, indem es Stadium 4 verlängert. Es hat aber keinen Einfluss auf Stadium 2 oder 3 und verzögert auch nicht generell die Progression der Erkrankung. Laut der Arbeit zeigt Riluzol dabei keine funktionellen Effekte, führt also bei ALS-Patienten im letzten Stadium zu einer Verlängerung der Lebenserwartung ohne begleitende Funktionsverbesserung. Ob die Behandlung mit Riluzol das Stadium 1 der Erkrankung beeinflusst, konnte in dieser Studie nicht untersucht werden.

Dr. Markus Numberger, Kandel 